\title{
EFFETS DE L'ECLAIRCIE SELECTIVE, PAR DEVITALISATION, SUR LA CROISSANCE EN EPAISSEUR DES ESSENCES PRINCIPALES DANS LA FORET CLASSEE DE BOSSEMATIE (CÔTE D'IVOIRE)
}

\author{
K. KOUADIO, K. E. KOUASSI, N.F.KOUAME et D.TRAORE
}

Laboratoire de Botanique, U.F.R. Biosciences, Université de Cocody-Abidjan 22 bp 582 Abidjan 22, Côte d'Ivoire. E-mail : attowoula@yahoo.fr

\begin{abstract}
RESUME
L'étude a été réalisée dans la forêt classée de Bossematié. Elle vise à évaluer les effets de l'éclaircie sélective, par dévitalisation, sur la croissance en épaisseur des tiges d'espèces principales d'arbres, dix ans après application d'un traitement sylvicole. La méthode de surface a été utilisée, pour la collecte des données. Les résultats montrent que la zone éclaircie et la zone non éclaircie ont présenté des indices de Shanon et d'équitabilité similaires (environ 7.41 et 0.94 , respectivement). Pour toutes les catégories confondues des essences principales, des densités moyennes de 283.7 et de 255.2 tiges/ha ont été obtenues pour la zone non éclaircie et éclaircie, respectivement pour les tiges moyennes. Quant aux grosses tiges, les densités ont été plus faibles (11.2 et 13.2 tiges/ha, respectivement). Dans toutes les classes de diamètre, l'analyse de variance a montré que les densités moyennes de la catégorie P1 ont été statistiquement égales dans les deux zones. Par contre, pour la catégorie P2, l'éclaircie sélective, par dévitalisation, a entraîné une baisse de densité de 16 tiges ha-1 dans la zone éclaircie contre 26.2 tiges/ha dans la zone non éclaircie. Pour la catégorie P3, au contraire, l'éclaircie a entraîné une augmentation significative de la densité (21.2 tiges/ha), contre 12.60 tiges/ha dans la zone non éclaircie). II apparaît donc que la dévitalisation n'a pas eu un effet significatif sur les tiges de la catégorie P1, de bonne qualité. Elle n'a entraîné que l'accroissement de densité des tiges de la catégorie P3, de moindre qualité.
\end{abstract}

Mots-clés : Forêt, sylviculture, essences commerciales, diversité floristique, densité.

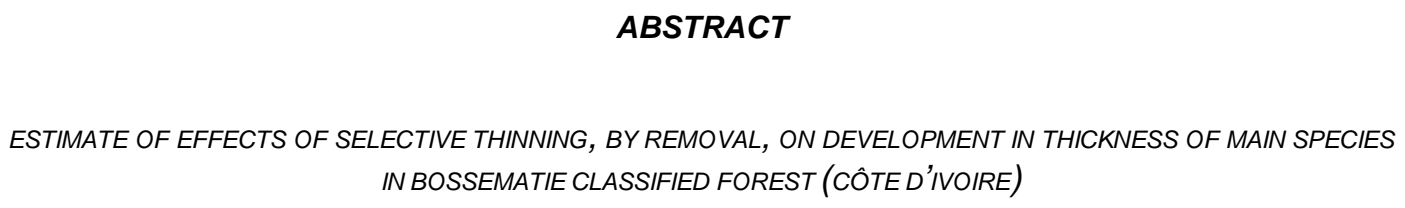

The study was undertaken at the Bossematie classified forest to evaluate the effects of selective thinning, by removal, on the development of stems of the tree main species, ten years after a syvicultural treatment. The plot method was used, as a mean for collecting data. Results show that average tree densities of both thinned and no thinned areas had similar Shannon and equitability indices (approximately 7.41 and 0.94 , respectively). For all categories, of the main tree species, average densities of 283.7 and 255.2 stems were obtained for both thinned and non thinned areas, respectively, with respect to the average-size stems. Regarding the larger-diameter stems, densities were much lower (11.2 and 13.2 stems ha ${ }^{-1}$, respectively). Within all classes of diameters, the analysis of variance shows that the $P 1$ category were somewhat equal in both areas. On the contrary, the $P 2$ category, where selective thinning, through stand removal, had resulted in a lower stand density in the thinned area (16 stems ha ${ }^{-1}$ ) against 26.2 stems ha ${ }^{-1}$ in the no thinned area. Conversely, for the P3 category, selective thinning has resulted in a significant increase in stems

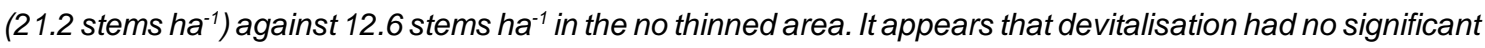
effect on the stems of $P 1$, of best quality. It resulted in the increase in the P3 category, of lowest category.

Key-words : Forest, silviculture, main species, floristic diversity, density. 


\section{INTRODUCTION}

La forêt constitue un écosystème en perpétuel renouvellement, du fait des processus de mortalité et de régénération. Ces derniers sont amplifiés par l'apparition de trouées dans le couvert végétal forestier (Dupuy et al., 1997) dont les origines sont diverses (exploitation forestière, éclaircies, incendies, défrichements, etc.). Le micro-climat forestier est ainsi périodiquement modifié par ces accidents naturels ou provoqués par l'homme (Hawthorne, 1994). La compréhension de la dynamique de la forêt naturelle, engendrée par ces accidents, passe par le suivi du peuplement adulte et de la régénération naturelle. En effet, les modifications des conditions micro-climatiques (lumière, température, humidité, etc.) consécutives à la disparition de certains arbres engendrent, d'une part, une réactivation de la croissance du peuplement sur pied et, d'autre part, l'apparition de nouveaux individus dans la régénération naturelle. Les ouvertures dans le couvert forestier, naturelles ou artificielles, ont une influence notable sur la dynamique de croissance du peuplement arbustif (Rivière, 1992).

La Société de Développement des Forêts (SODEFOR) en Côte d'Ivoire, chargée de la gestion depuis 1992, des 231 forêts classées, a réparti les espèces arborées ligneuses en deux groupes (essences principales et essences secondaires) en fonction de leur valeur technologique évaluée dans un objectif de bois d'œuvre (Dupuy et al., 1997). Les essences principales sont des espèces commercialisées, ou à promouvoir du fait de leurs bonnes caractéristiques technologiques. Elles sont subdivisées en trois catégories selon leurs qualités (Anonyme, 1989). Les espèces de la catégorie P1 sont de meilleure qualité ; celles de la catégorie $\mathrm{P} 2$ sont de qualité moyenne et celles de la catégorie $\mathrm{P} 3$ sont de moindre qualité. Les essences secondaires sont les espèces qui ne sont pas commercialisées, à ce jour, en bois d'œurre.

C'est essentiellement au profit des essences principales que l'éclaircie sélective, par dévitalisation, a été réalisée dans la forêt classée de Bossematié (N'cho, 2000a), depuis 1992. La réalisation de l'éclaircie sélective, par dévitalisation, dans cette forêt classée, découle des résultats obtenus suite aux expérimentations faites dans les forêts classées de
Irobo, Mopri et Téné par la SODEFOR et CIRADforêt en 1977-1978. Ces expérimentations qui ont porté sur l'accroissement en épaisseur des essences principales forestières ont montré un effet net des éclaircies (moyenne et forte) sur l'accroissement en épaisseur des essences principales dans la parcelle traitée, par rapport au témoin. Mais, il n'y a pas eu de différence significative entre l'éclaircie moyenne et l'éclaircie forte (Maître et Hermeline, 1985 ; kouassi, 2000). C'est donc dans le but de vulgariser les résultats acquis, dans un objectif de développement, que cette opération a été menée dans la forêt classée de Bossematié.

Les ouvertures créées dans la forêt classée de Bossematié par l'application de l'éclaircie sélective par dévitalisation, dix années après, ont-elles profité aux tiges des essences principales? En d'autres termes, la croissance en épaisseur de ces tiges a-t-elle été plus rapide dans les parcelles éclaircies que dans les parcelles non éclaircies ? Quelles sont les catégories d'espèces principales dont les tiges ont été favorisées par ce traitement sylvicole?

L'objectif principal de cette étude est d'évaluer l'influence de l'éclaircie sélective, par dévitalisation, sur la croissance en épaisseur des tiges de plus de $10 \mathrm{~cm}$ d.b.h. des essences principales. Comme objectifs spécifiques, il s'agit de :

- déterminer la diversité spécifique et la distribution des tiges d'espèces dans la zone éclaircie de même que dans la zone non éclaircie ;

- comparer les densités moyennes des groupes d'essences au sein des classes de diamètre, dans les deux zones d'étude.

\section{MATERIEL ET METHODES}

\section{MATERIEL}

L'étude a été réalisée dans la forêt classée de Bossematié, située dans le secteur mésophile du Domaine guinéen, dans l'Est de la Côte $d^{\prime} I v o i r e\left(6^{\circ} 20^{\prime}\right.$ et $6^{\circ} 35^{\prime} \mathrm{N}$, et $3^{\circ} 20^{\prime}$ et $\left.3^{\circ} 35^{\prime} \mathrm{W}\right)$, à $30 \mathrm{~km}$ au Sud-Est de la ville d'Abengourou (Figure 1). Elle occupe une superficie de 21553 ha (Anonyme, 1994). La zone est caractérisée par une température moyenne annuelle de $26,5^{\circ} \mathrm{C}$ de 1986 à 1994 et par une pluviométrie moyenne annuelle sur les neuf ans, 


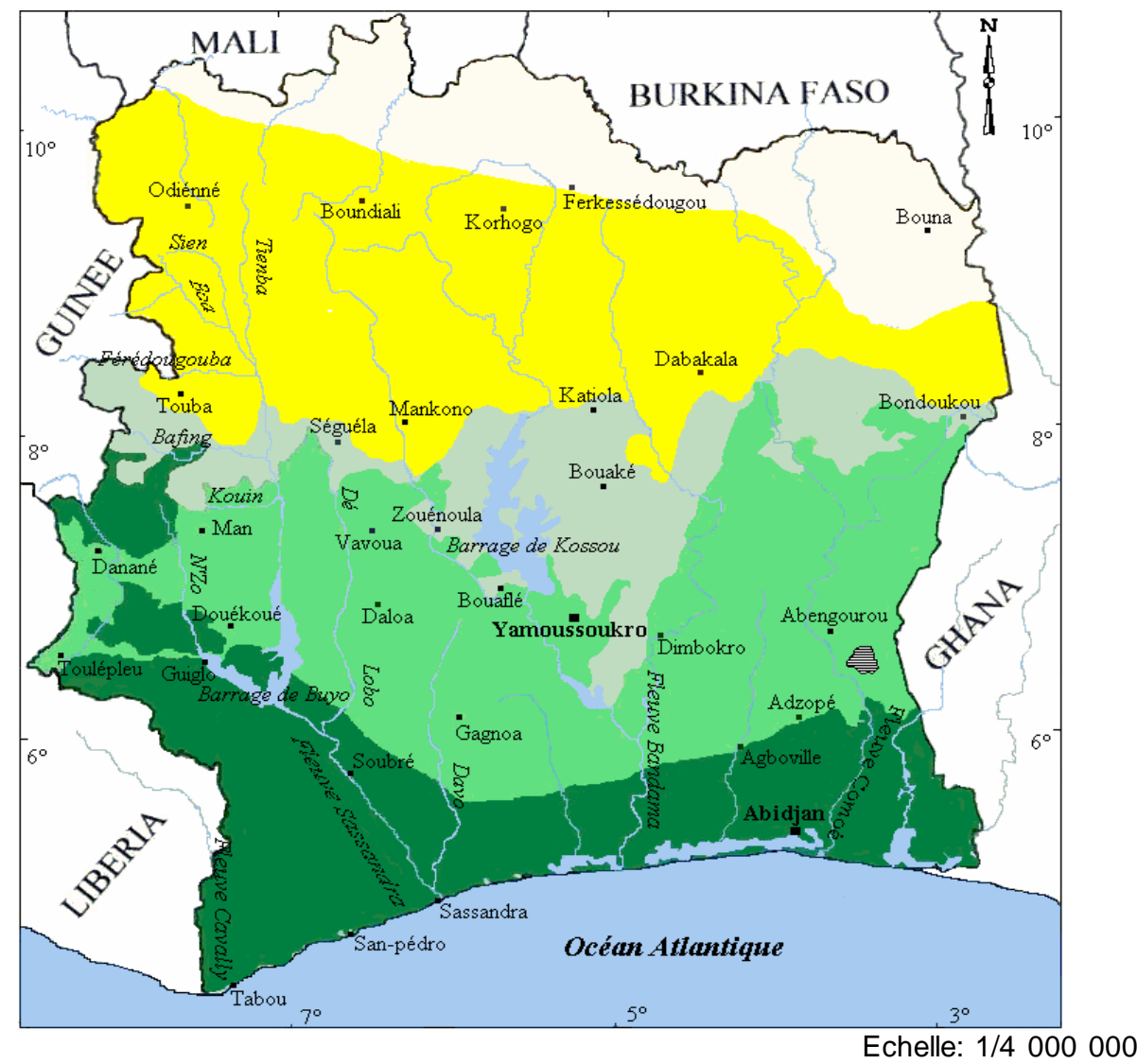

Domaine guinéen

Secteur ombrophile

Secteur mésophile

Secteur préforestier

Forêt classée de Bossematié
Domaine soudanais

Secteur subsoudanais

Secteur soudanais

Figure 1 : Localisation de la forêt classée de Bossematié en Côte d'Ivoire (Source : Monnier, 1983).

Location of Bossematié classified forest in Côte d'Ivoire (Source : Monnier, 1983).

de 1247,21 mm (Bakayoko, 1999). Les sols les plus dominants sont les ferrasols (Anonyme, 2002). La végétation est du type semi-décidue (Guillaumet et Adjanohoun,1971). Cette végétation est composée de formations à Celtis spp. et de Triplochiton scleroxylon au Nord, de Khaya spp. et de Nesogordonia papaverifera au Sud (Anonyme, 2002).

L'application de l'éclaircie sélective, par dévitalisation dans cette forêt classée, se situe dans le cadre de l'amélioration de la productivité en bois d'œuvre des forêts naturelles ivoiriennes (Anonyme, 1998). Cette technique sylvicole a consisté à faire périr, sur pied, des tiges d'essences secondaires de plus de $10 \mathrm{~cm}$ d.b.h., en vue de faciliter la croissance en épaisseur des tiges d'essences principales. L'opération a été effectuée sur une superficie de 800 ha qui constitue la zone éclaircie et 200 ha qui représentent la zone non éclaircie, consécutive à celle éclaircie.

Le matériel biologique utilisé a été constitué de tiges d'espèces principales. 


\section{METHODES}

\section{Dévitalisation dans la forêt classée de Bossematié}

La dévitalisation a été réalisée selon deux méthodes (Fickinger, 1995), notamment :

- l'annelation qui a consisté en un arrachage de l'écorce autour des tiges sur une hauteur de 15 à $20 \mathrm{~cm}$ avec une hachette, pour les arbres de petits diamètres, dans le but d'arrêter la
- et la réalisation de deux entailles profondes, jusqu'au cœur des tiges (sur les deux côtés opposés), à l'aide d'une tronçonneuse pour les arbres de gros diamètres de sorte à interrompre la circulation de la sève.

\section{Collecte des données}

Pour mesurer les diamètres des arbustes et arbres de plus de $10 \mathrm{~cm}$ d.b.h., nous avons disposé sur le terrain, un placeau de 4 ha, de forme rectangulaire, de $400 \mathrm{~m}$ de longueur et

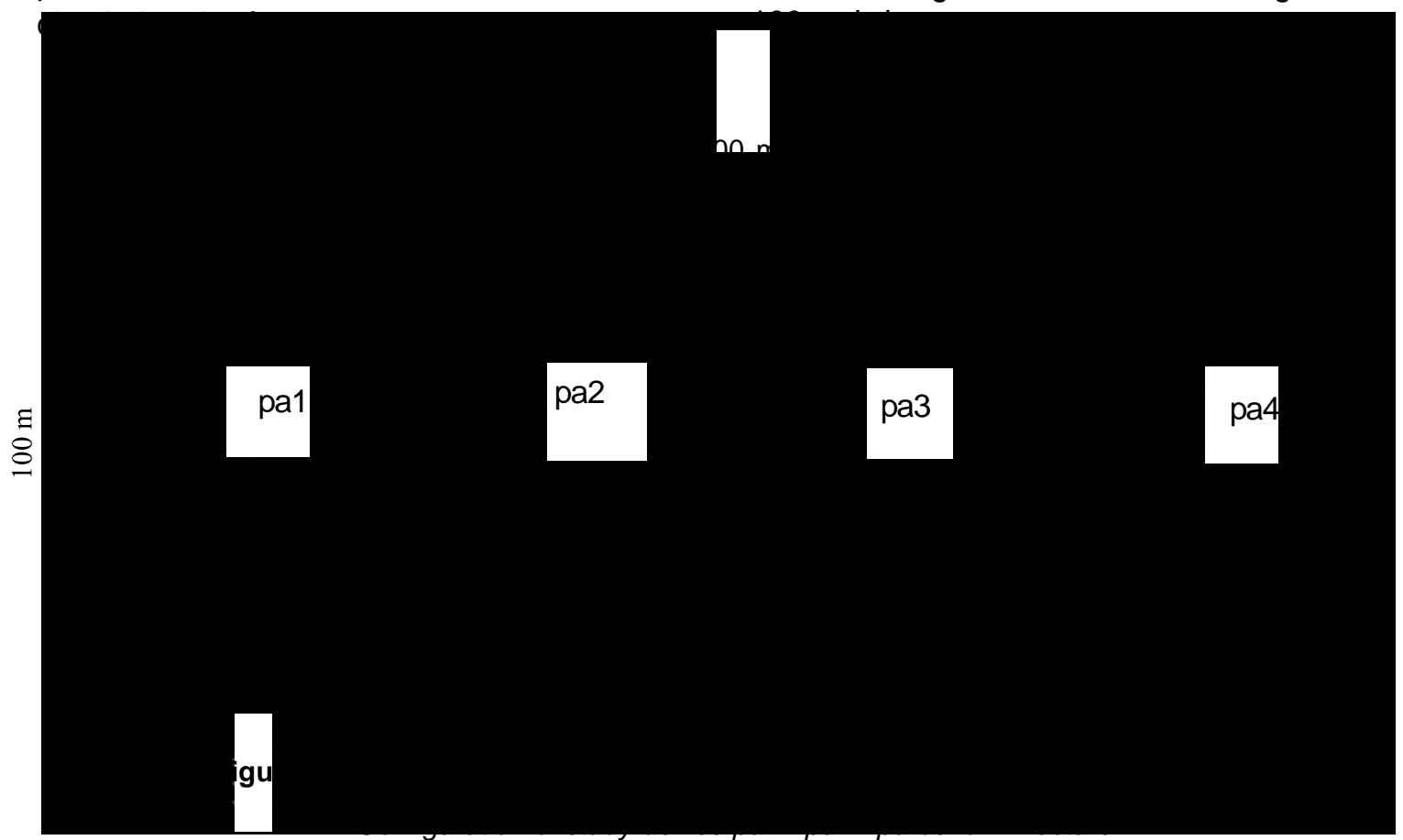

Celui-ci a été subdivisé en quatre parcelles (pa1 à pa4) de 1 ha chacune, de forme carrée, de $100 \mathrm{~m}$ de côté (Figure 2). Dix ont été installé dans la forêt classée, dont cinq placeaux dans la zone non éclaircie et cinq autres dans la zone éclaircie. Au total, 40 parcelles de 1 ha chacune ont été installées, dont 20 dans la zone éclaircie et 20 autres dans la zone non éclaircie. Pour réduire les erreurs d'échantillonnage, chaque parcelle a été subdivisée en 100 sous-parcelles (sp) de $100 \mathrm{~m}^{2}$ chacune, de forme carrée, de 10 $\mathrm{m}$ de côté (Figure 3 ). Dans chaque sous parcelle, nous avons identifié et mesurer à l'aide de rubans dendrométriques les diamètres de tous les arbustes et arbres de plus de $10 \mathrm{~cm} \mathrm{d.b.h.} \mathrm{Chaque} \mathrm{tige} \mathrm{mesurée}$ est marquée à la peinture à huile.

\section{Méthodes d'analyse}

\section{Diversité floristique et indice d'équitabilité}

Les indices de Shannon et Weaver ( $\mathrm{H}^{\prime}$ ) et d'E (E) ont été utilisés pour déterminer la diversité floristique et la distribution équitable des individus des espèces dans les deux zones d'étude, dix ans après l'éclaircie. L'indice de Shannon et Weaver est exprimé comme suit :

$$
\begin{aligned}
& \mathrm{N}
\end{aligned}
$$

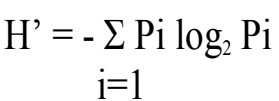

où $\mathrm{N}$ effectif des $\mathrm{S}$ espèces considérées, ni l'effectif des individus d'une espèce i et $\mathrm{Pi}=$ ni/ $\mathrm{N}$ l'abondance relative de l'espèce $\mathrm{i}$. 
L'indice d'équitabilité (Piélou, 1966), appelé également indice d'équirépartition a été estimé par :

$$
\mathrm{E}=\mathrm{H}^{\prime} / \log _{2} \mathrm{~S}
$$

Les valeurs des deux indices de la zone éclaircie ont été comparées à celles de la zone témoin.

\section{Analyse de variance}

Au sein des classes de diamètres, les densités moyennes des différentes catégories d'essences principales de la zone éclaircie ont été comparées à celles obtenues dans la zone non éclaircie, à travers l'analyse de variance à un facteur, ANOVA1 définit par Dagnélie (1999). Ces analyses de variance ont été réalisées en utilisant le Test de Newman-Keuls, avec un seuil de probabilité fixé à $\mathrm{P} \leq 0,05$ (Tableau 1 ).

\section{RESULTATS}

\section{DIVERSITE FLORISTIQUE ET DISTRIBUTION DES INDIVIDUS D'ESPECES DANS LES DEUX ZONES D'ETUDE}

Dans la zone non éclaircie, les valeurs des indices de Shannon et d'équitabilité sont, respectivement de 7,41 et de 0,94. Dans la zone éclaircie, la valeur de l'indice de Shannon est de 7,37 et celle de l'indice d'équitabilité est de 0,94 .

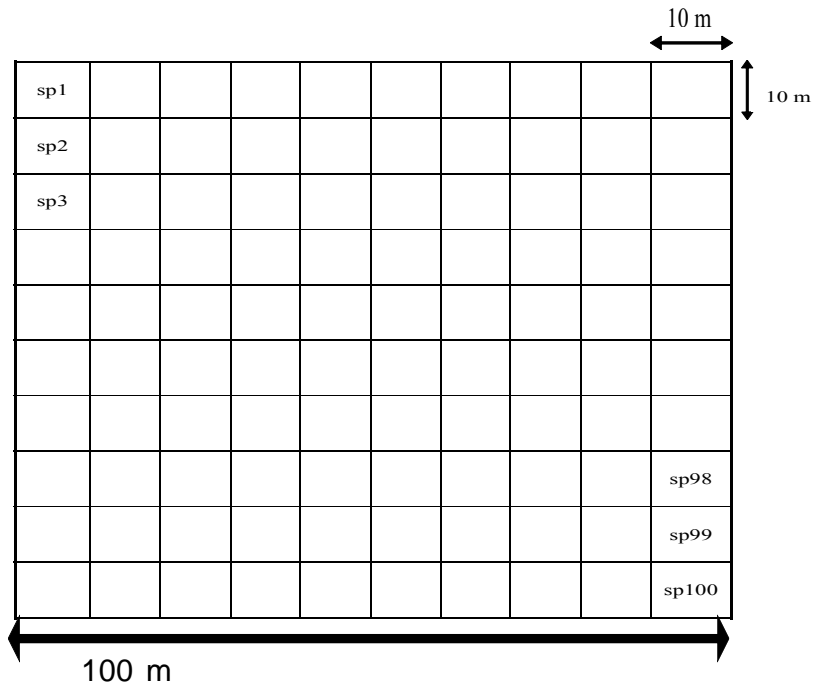

Figure 3 : Configuration des sous parcelles d'étude sp1-sp100: sous parcelle de $100 \mathrm{~m}^{2}$ layout of plots sp $100\left(100 \mathrm{~m}^{2}\right)$.

Tableau 1 : Répartition des densités initiales des différentes catégories des essences principales, au sein des classes de diamètres, dans la forêt classée de Bossematié (source : Anonyme, 1994).

Distribution of the initial densities of the various categories of main tree species, within the classes of diameters, in Bossematié classified forest.

\begin{tabular}{lcccc}
\hline & \multicolumn{4}{c}{ Densités (tiges/ha) } \\
\cline { 2 - 5 } Classes de diamètres $(\mathrm{cm})$ & $\mathrm{P} 1$ & $\mathrm{P} 2$ & $\mathrm{P} 3$ & Total \\
\hline$[10 ; 20[$ & 44,9 & 49,2 & 8,5 & 102,6 \\
{$[20 ; 30[$} & 13,9 & 16,1 & 3,9 & 33,9 \\
{$[30 ; 40[$} & 10,1 & 6,1 & 2,5 & 18,7 \\
{$[40 ; 50[$} & 6,3 & 3,6 & 1,2 & 11,1 \\
{$[50 ; 60[$} & 1,9 & 2,5 & 0,6 & 5,0 \\
{$[60,+\infty[$} & 2,0 & 3,0 & 0,9 & 5,9 \\
\hline Tiges moyennes & 77,1 & 77,5 & 16,7 & 171,3 \\
$(10 \mathrm{~cm} \leq$ diamètres $<60 \mathrm{~cm})$ & 2,0 & 3,0 & 0,9 & 5,9 \\
\hline Grosses tiges & & & & \\
$($ diamètres $>60 \mathrm{~cm})$ & & & & \\
\hline
\end{tabular}

$\mathrm{P}:$ Catégories des essences principales

$P$ : Categories of main species 


\section{DENSITES MOYENNES DES DIFFERENTES CATEGORIES D'ESSENCES PRINCIPALESAU SEIN DES CLASSES DE DIAMETRES}

L'analyse du tableau 2 donne 283,7 tiges/ha d'essences principales, toutes catégories confondues, dans la zone non éclaircie et 255,2 tiges/ha de celles-ci dans la zone éclaircie de tiges moyennes. Quant aux grosses tiges, les densités de ces essences, sont de 11,2 tiges/ha pour la zone non éclaircie et de 13,2 pour la zone éclaircie.

Dans les classes de diamètres de $[10 ; 20$ [à $[60,+\infty$ [, les densités moyennes de la catégorie P1 des essences principales, sont statistiquement égales, entre la zone non éclaircie et la zone éclaircie (Tableau 3). Dans la classe [20 ; 30[, la densité moyenne de 26,20 tiges/ha de la catégorie $\mathrm{P} 2$ de la zone non éclaircie, est statistiquement différente de celle
(16,00 tiges/ha) trouvée dans la zone éclaircie (Tableau 3). Cette différence entre ces densités moyennes est très significative $(p=0,006)$. Quant aux densités moyennes des deux zones d'étude de cette catégorie d'essences principales, dans les autres classes de diamètres, elles sont invariables statistiquement (Tableau 3). Concernant, la catégorie P3, la densité moyenne de 21,20 tiges/ha de la zone éclaircie est statistiquement différente (Tableau 3) de celle (12,60 tiges/ha) obtenue dans la zone non éclaircie, dans la classe [10; 20[. De même, cette différence statistique des densités moyennes (Tableau 3) est, également, relevée dans la classe [50;60[, pour la même catégorie d'essences principales. Ces différences entre ces densités moyennes des deux zones d'étude avec des probabilités de 0,011 et de 0,046 (Tableau 3) ont été obtenues, respectivement, dans les classes $[10 ; 20[$ et $[50 ; 60[$.

Tableau 2 : Comparaisons des densités (tiges/ha) des différentes catégories d'essences principales des deux zones d'étude aux densités initiales de ces essences au sein des classes de diamètres.

Comparisons of average densities (stems/ha) of the various categories of main species of two area of study with the initial densities of these species, within the classes of diameters.

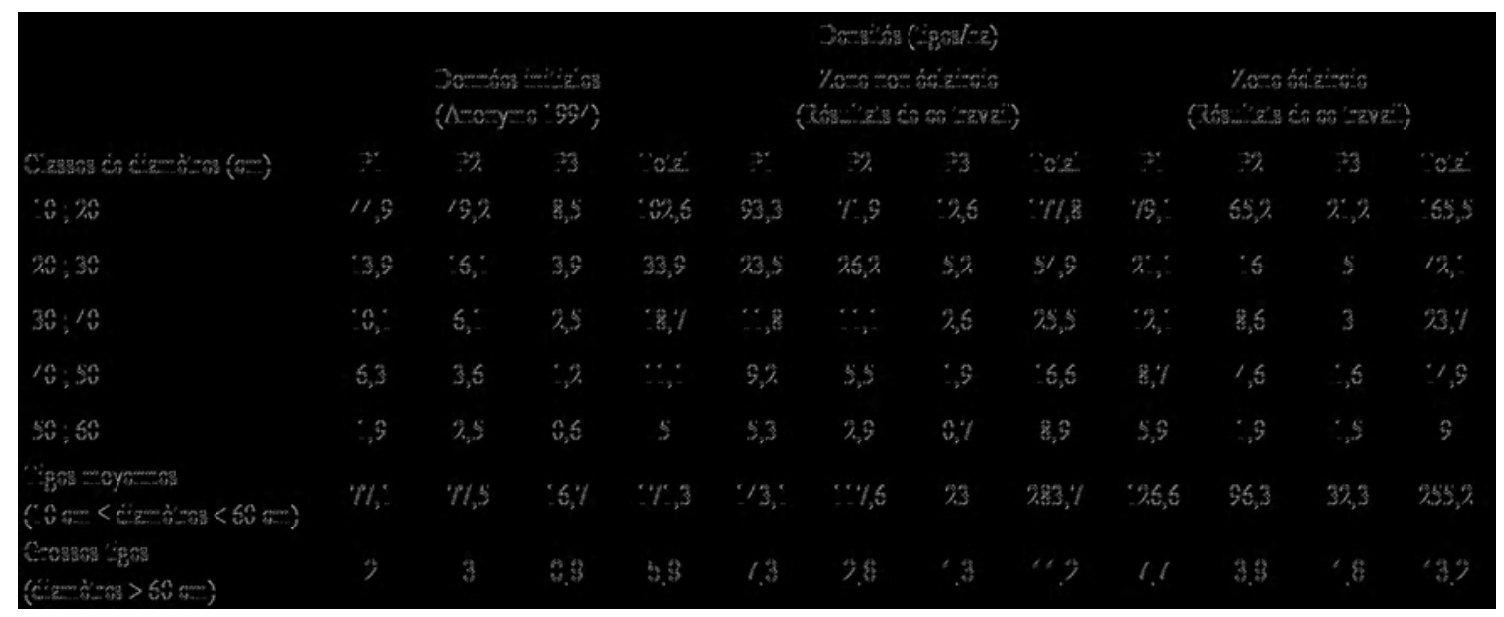

P: Catégories d'essences principales

$P$ : Categories of main species 
Tableau 3 : Comparaisons des densités moyennes des différentes catégories d'essences principales, parl'ANOVA1, dans les deux zone d'étude, au sein des classes de diamètres.

Comparisons of the average densities of the various categories of main species, by ANOVA1,

\begin{tabular}{|c|c|c|c|c|c|c|c|c|c|c|c|c|}
\hline \multirow[b]{2}{*}{$\begin{array}{c}\text { Classes de } \\
\text { diamètres } \\
(\mathrm{cm})\end{array}$} & \multicolumn{4}{|c|}{ Catégories P1 des essences principales } & \multicolumn{4}{|c|}{ Catégories P2 des essences principales } & \multicolumn{4}{|c|}{ Catégories P3 des essences principales } \\
\hline & $\begin{array}{c}\text { Zone non } \\
\text { éclaircie }\end{array}$ & $\begin{array}{l}\text { Zone } \\
\text { éclaircie }\end{array}$ & Probabilités & Résultats & $\begin{array}{c}\text { Zone non } \\
\text { éclaircie }\end{array}$ & $\begin{array}{l}\text { Zone } \\
\text { éclaircie }\end{array}$ & Probabilités & Résultats & $\begin{array}{c}\text { Zone non } \\
\text { éclaircie }\end{array}$ & $\begin{array}{l}\text { Zone } \\
\text { éclaircie }\end{array}$ & Probabilités & Résultats \\
\hline$[10 ; 20[$ & 90,3 & 79,1 & 0,35 & NS & 71,85 & 65,15 & 0,44 & NS & 12,6 & 21,2 & 0,011 & S \\
\hline$[20 ; 30[$ & 23,5 & 21,05 & 0,52 & NS & 26,2 & 16 & 0,006 & $\mathrm{~S}$ & 5,2 & 5 & 0,83 & NS \\
\hline$[30 ; 40[$ & 11,8 & 12,05 & 0,86 & NS & 11,1 & 8,6 & 0,22 & NS & 2,6 & 3 & 0,55 & NS \\
\hline$[40 ; 50[$ & 9,15 & 8,7 & 0,67 & NS & 5,45 & 4,55 & 0,43 & NS & 1,85 & 1,6 & 0,67 & NS \\
\hline$[50 ; 60[$ & 5,3 & 5,55 & 0,81 & NS & 2,9 & 1,9 & 0,13 & NS & 0,7 & 1,5 & 0,046 & $\mathrm{~S}$ \\
\hline$[60,+\infty[$ & 3,95 & 3,8 & 0,85 & NS & 1,6 & 1,7 & 0,81 & NS & 0,75 & 0,6 & 0,58 & NS \\
\hline
\end{tabular}

S: Significativité, NS: Non Significativité

S: Significant, NS: No Significant

\section{DISCUSSION}

\section{DIVERSITE FLORISTIQUEET DISTRIBUTION DES INDIVIDUS D'ESPECES DANS LES DEUX ZONES D'ETUDE}

Les valeurs de l'indice de Shannon et Weaver sensiblement égales dans la zone non éclaircie et dans la zone éclaircie, montre que ces deux milieux d'étude ont une diversité floristique, quasiment invariable. La dévitalisation des tiges en faveur de celles de la catégorie P1 des essences principales, dans la zone éclaircie n'a eu aucun effet sur la diversité floristique de ce milieu. En revanche, dans la zone éclaircie comme dans la zone non éclaircie, les valeurs de l'indice d'équitabilité ont été identiques et proches de 1 ; ceci permet de dire que les espèces rencontrées dans chacune des zones d'étude ont la même abondance. Ainsi, les individus de ces espèces sont équitablement répartis.

\section{DENSITES MOYENNES DES DIFFERENTS GROUPES D'ESSENCES AU SEIN DES CLASSES DE DIAMETRES}

Pour les tiges moyennes et les grosses tiges, les densités obtenues dans la zone non éclaircie et dans la zone éclaircie, sont des densités initiales. Cette augmentation de tiges dans les deux milieux d'étude par rapport aux données initiales, montre une dynamique de croissance en épaisseur des essences principales, dix ans après l'intervention sylvicole. Cependant, pour les tiges moyennes, la densité a été plus élevée dans la zone non éclaircie que dans la zone éclaircie. Cela est due, soit à la dévitalisation des tiges réalisée dans la zone éclaircie, soit, au chablis empêchant le développement des jeunes tiges, de moins de $10 \mathrm{~cm}$ d.b.h. comme l'a souligné Bertauld (1986). Par contre, la tendance a été inversée au niveau des gains en grosses tiges dans les deux zones d'étude. En effet, la densité de grosses tiges des essences principales, a été relativement élevée dans la zone éclaircie par rapport à la zone non éclaircie. Ceci confirme la dynamique de croissance en épaisseur des espèces principales. Au niveau des grosses tiges, la croissance en épaisseur a semblé plus rapide dans la zone éclaircie que dans la zone non éclaircie. Cependant, il n'y a pas de eu différence significative entre les densités de la zone non éclaircie et celles de la zone éclaircie.

La différence significative, entre les moyennes de la catégorie $\mathrm{P} 2$, relevée dans la classe [20; 30[, indique que le nombre de tiges de plus de $10 \mathrm{~cm}$ d.b.h. de ces espèces principales, a été plus élevé dans la zone non éclairci, que dans la zone éclaircie. Cette faible densité de ces tiges, obtenue dans la zone éclaircie est due à la dévitalisation des individus de la catégorie P2 au profit de ceux de la catégorie P1. Par conséquent, l'effectif du peuplement arbustif de la catégorie $\mathrm{P} 2$ a été fortement réduit dans la zone éclaircie. Selon Fickinger (1995), les tiges des catégories $\mathrm{P} 2$ et $\mathrm{P} 3$ et celles des essences secondaires, des classes de diamètres [20 ; $30[$, [30; 40[ et [40;50[ ont été les plus dévitalisées. En soutenant la réduction du 
nombre de tiges, dans la zone éclaircie, cet auteur (I.c.) a souligné que, deux ans après l'application du traitement, le taux de mortalité des individus dévitalisés s'est élevé à $85 \%$. C'est pourquoi, dans le suivi de l'éclaircie sélective, par dévitalisation, N'cho (2000b) préconise, la non dévitalisation d'une essence principale au profit d'une autre, quelle que soit sa catégorie et sa conformation. Si cette recommandation est rigoureusement appliquée, la mortalité des tiges des espèces principales sera réduite de façon significative. Dans les classes [10;20[ et [50;60[, les effectifs des tiges de plus de 10 $\mathrm{cm}$ d.b.h. de la catégorie $\mathrm{P} 3$ ont été plus élevés dans la zone éclaircie que ceux obtenus dans la zone non éclaircie. Quoique certaines de ces tiges aient été dévitalisées, leur nombre a considérablement augmenté dans la zone éclaircie, au niveau de ces deux classes de diamètres. Les fortes densités moyennes de la catégorie $\mathrm{P} 3$ relevées dans les classes $[10$; 20[ et [50;60[, de la zone éclaircie, par rapport à celles de la zone non éclaircie, attestent de l'effet bénéfique du traitement sylvicole sur cette catégorie d'espèces principales. L'éclaircie sélective, par dévitalisation, a favorisé donc l'augmentation des tiges dans les classe [10; 20 [ et [50;60[. L'augmentation du nombre de tiges de la catégorie P3, dans la classe $[10$; 20[, montre que le traitement sylvicole a facilité davantage la croissance de la régénération naturelle de cette catégorie d'espèces principales dans la zone éclaircie. Durrieu et al. (1998) ont fait les mêmes observations dans les dispositifs expérimentaux de Mopri. Pour la catégorie P3, l'éclaircie sélective, par dévitalisation, a donc induit aux jeunes plants de moins de $10 \mathrm{~cm}$ d.b.h. une croissance en épaisseur et en hauteur plus rapide, leur permettant d'atteindre et même de dépasser le diamètre précomptable qui est de $10 \mathrm{~cm}$. En effet, les ouvertures créées dans le couvert végétal par la chute des tiges dévitalisées et mortes, ont permis à la lumière d'atteindre facilement les jeunes plants. Cette lumière a donc stimulé la croissance de ces jeunes plants. Meer (1995) en confirmant l'action stimulante de la lumière sur la croissance en épaisseur et en hauteur des jeunes tiges, a souligné que l'ouverture de la canopée et la disponibilité de la lumière sont des facteurs favorisant la croissance rapide de celles-ci.

Cependant, il relativise son idée et affirme que la vitesse de croissance de ces jeunes plants dépend des caractéristiques de chaque espèce. Sterck (1997) soutient cette idée et indique que les jeunes tiges en milieu lumineux grandissent plus rapidement que dans un milieu ombragé. Dans la forêt classée d'Irobo, Brevet et Diahuissie (1993) ont mentionné l'effet bénéfique de l'éclaircie sur le peuplement d'essences principales et ont relevé que les tiges de la catégorie P2 ont été plus favorables au traitement sylvicole que celles des catégories P1 et P3. Dans la forêt classée de Bossematié, notre étude montre que ce sont les individus des P3 qui ont plus profité de l'éclaircie sélective, par dévitalisation.

\section{CONCLUSION}

Cette étude a montré que l'éclaircie sélective, par dévitalisation, n'a eu aucun effet sur la diversité floristique. En revanche, les tiges de plus de $10 \mathrm{~cm}$ d.b.h. des différentes espèces rencontrées sont équitablement réparties dans les deux milieux d'étude.

Contrairement, aux catégories $\mathrm{P} 1$ et $\mathrm{P} 2$ des essences principales, l'éclaircie sélective, par dévitalisation, a favorisé, seulement, l'augmentation des individus de plus de $10 \mathrm{~cm}$ d.b.h. de la catégorie P3, dans la zone éclaircie, dix années après l'application du traitement sylvicole.

Cette réaction «défavorable» des tiges des catégories P1 et P2 face à l'éclaircie, est liée au choix des tiges dévitalisées et probablement, aussi, au suivi technique de l'éclaircie sélective, par dévitalisation.

\section{REFERENCES}

Anonyme 1989. Momento du forestier. Minist. Coopérat. Dével., Paris, 1266 p.

Anonyme 1994. Plan d'aménagement de la forêt classée de la Bossematié. SODEFOR, Abengourou, Rapport d'activité, $85 \mathrm{p}$.

Anonyme 1998. Plan Directeur Forestier 19882015. Ministère des Eaux et Forêt, Doc. technique : pp 1- 5.

Anonyme 2002. Plan d'aménagement de la forêt classée de la Bossematié, SODEFOR, Abengourou, Rapport d'activité, 120 p.

Bakayoko A. 1999. Comparaison de la composition floristique et de la structure forestière de parcelles de la forêt classée de Bossematié, dans l'Est de la Côte d'Ivoire. Mémoire de DEA, UFR Biosciences, Université d'Abidjan-Cocody (Côte d'Ivoire), $72 \mathrm{p}$. 
Bertauld J. G. 1986. Etude de l'effet d'interventions sylvicoles sur la régénération naturelle au sein d'un périmètre expérimental d'aménagement, en forêt dense humide de Côte-d'Ivoire. Thèse de I'Université de Nancy (faculté des sciences), 254 p.

Brevet R. et A. Diahuissie. 1993. Étude des traitements sylvicoles en forêt sempervirente : comparaison des résultats obtenus à 4 ans dans le massif de Yapo et le périmètre expérimental d'Irobo, IDEFOR/ DFO. Côte d'Ivoire, Rapport d'étude, Abidjan, $46 \mathrm{p}$.

Dagnélie P. 1999. Théories et méthodes statistiques Tome 2, Presse agronomique de Gembloux, 463 p.

Dupuy B., F. Doumbia, A. Diahuissié et R. Brevet. 1997. Effet de types d'éclaircie en forêt dense humide ivoirienne. Bois et Forêt Tropiques 253 (3) : 5 - 18.

Durrieu L. M., V. Fabrichon, B. Dupuy, A. Barhen, L. Houde et H. F. Maître. 1998. Croissance et production en forêt dense humique : bilan des expérimentations dans le dispositif de Mopri-Côte d'Ivoire (1978 - 1992), CIRADForêt, Paris, $73 \mathrm{p}$.

Fickinger H. 1995. Les effets de l'éclaircie sélective en forêt classée de la Bossematié sur l'accroissement des arbres d'avenir. SODEFOR/GTZ, Rapport d'étude, Abengourou, $6 \mathrm{p}$.

Guillaumet J. L. et E. Adjanohoun. 1971. La végétation, In : Avenard J. M., Eldin E., Sircoulou J., Touchebeuf P., Guillaumet J. L., Adjanohoun E. et Perraud A. (Eds.). Le milieu naturel de la Côte d'Ivoire, Mém. ORSTOM 50 : 161 - 262.

Hawthorne W. D. 1994. Fire damage and forest regeneration in Ghana. London, UK, Forestry series 4, $53 \mathrm{p}$.

Kouassi R. H. 2000. Contribution à l'étude de l'accroissement en diamètre d'essences forestières au sein de trois périmètres expérimentaux : Mopri, Téné et Irobo (Côted'Ivoire). Mémoire de DEA, UFR Biosciences, Université d'Abidjan-Cocody (Côte d'Ivoire), 64 p.

Maître H. et M. Hermeline. 1985. Dispositif d'étude de l'évolution de la forêt dense ivoirienne suivant différentes modalités d'intervention sylvicole. Présentation des principaux résultats après 4 années d'expérimentation, $73 \mathrm{p}$.

Meer P. J. V. D. 1995. Canopy dynamics of a tropical rain forest in French Guiana. PhD thesis, Wageningen Agricultural University, The Netherlands, 149 p.

Monnier Y. 1983. Carte de la végétation de la Côte d'Ivoire. In : Vennetier P. et Laclavère G., Eds. Atlas de Côte d'Ivoire $2 e$ ed. Paris, France : Jeune Afrique, $72 \mathrm{p}$.

N'cho D. A. 2000a. Évaluation de l'impact d'une éclaircie sélective par dévitalisation sur des peuplements forestiers naturels des forêts classées de la Niégré et du HautSassandra. Mémoire de fin de cycle d'Ingénieur. INPHB/ECO.SYN/SODEFOR, Yamoussoukro, Côte d'Ivoire, 83 p.

N'cho D. A. 2000b. Sylviculture en forêt naturelle : protocole d'éclaircie sélective, par dévitalisation. SODEFOR Rapport d'activité, Abidjan, Côte d'Ivoire, 9 p.

Piélou E. C. 1966. The measurement of diversity in different types of biological collections. Journal of Theoretical Biology. vol 13 : 131144.

Rivière L. 1992. Etude de l'évolution des peuplements naturels d'Okoumé (Aucoumea klaineana) dans le sudestuaire du Gabon. Thèse Univ. Paris VI, France, 176 p.

Sterck F. J. 1997. Trees and light: tree development and morphology in relation to light availability in a tropical rain forest in French Guiana. Wageningen, 122 p. 